Rückwirkungen der internetgestützten Internationalisierung des Vertriebs auf die Abfallpolitik

\section{Produktverantwortung ausgehebelt}

\begin{abstract}
Der grenzüberschreitende Direkfvertrieb an inländische Endkunden insbesondere durch E-Commerce wirft ein gravierendes Problem für Rücknahmeverpflichtungen auf: Zunehmend können Produkte in die hiesigen Verwertungs- und Beseitigungsströme gelangen, bei denen es nicht gelingt, dem Hersteller oder Importeur die Produktverantwortung zuzuweisen. Dies kann ökologische Innovationen in diesem Bereich behindern. Auswege sind allerdings nicht einfach.
\end{abstract}

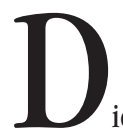

Von Hartmut Clausen und Marianne Halstrick-Schwenk schaftspolitik folgen dem Paradigma der Kreislaufwirtschaft und normieren eine Produktverantwortung der Hersteller bzw. Inverkehrbringer bestimmter Produktgruppen. Als wichtigstes Instrument dienen ihnen Rücknahmeverpflichtungen, mit denen eine Neuverteilung von Verfügungsrechten und -pflichten in Hinblick auf die Entsorgung von Produkten vorgenommen wird. Rücknahmeverpflichtungen können zwar nicht die Internalisierung negativer externer Effekte substituieren, sie sind aber geeignet, speziell in der Abfallwirtschaft auftretende Probleme - die Anreize zur Wahl des ,richtigen“ Entsorgungsweges und die so genannte Entkopplung abfallrelevanter Entscheidungen - anzugehen (1). Damit können Rücknahmeverpflichtungen helfen, die Anreiz- und Innovationswirkungen anderer Instrumente zur vollen Entfaltung zu bringen.

Die zunehmende und durch die Nutzung neuer Medien begünstigte Internationalisierung von Produktion und Nachfrage könnte jedoch zu einem ernst zu nehmenden Defekt führen. Gelangen Produkte über direkte und grenzüberschreitende Vertriebswege an Endverbraucher, gelingt die Zuweisung bzw. Durchsetzung von Produktverantwortung eventuell nicht mehr. Dies ist der Fall, wenn der Hersteller keine Niederlassung im Geltungsbereich der Verordnungen hat und kein Zwischenhandel über einen Importeur existiert. Anders als die EU-Richtlinien zur Rücknahme von Elektrogeräten und von Altautos ist die Europäische Verpackungsrichtlinie bereits durchweg in nationales Recht übertragen worden, weshalb ihr in Bezug auf die Berücksichtigung von Direktvertreibern eine Leiffunktion für andere Warengruppen zukommt. Hier zeigt sich, dass zwar eine Anwendung auf sämtliche in das jeweilige Gebiet ge-
Die für diesen Regelungsdefekt relevanten Handelsströme sind grenzüberschreitend und durch eine räumliche Trennung von Anbietern und Käufern gekennzeichnet (Direktvertrieb). Neben dem traditionellen Versandhandel trifft dies typischerweise auf den Electronic Commerce, den elektronisch unterstïtzten Handel mit Waren und Dienstleistungen, zu (3). Während beim Versandhandel der Kauf selbst durch schriftlichen oder telefonischen Auftrag und der Warenversand per Post oder Paketdienst erfolgt, werden hier Angebot und Bestellung durch ein den Online-Handel ermöglichendes Endgerät abgewickelt. Beratung, Auslieferung und Zahlungsabwicklung können auf traditionellem Wege oder elektronisch unterstïtzt durchgeführt werden. Für die Frage der Zuweisung von Produktverantwortung im Kontext von Rücknahmeverpflichtungen ist insbesondere der Warenhandel zwischen Unternehmen und Endverbrauchern von Interesse, das heißt der Businessto-Consumer-Bereich (B2C).

$\mathrm{Zu}$ den Anbietern gehören damit der Versandhandel im institutionellen Sinne, dem ein beträchtliches Wachstum beim grenzüberschreitenden Handel prognostiziert wird. Hinzu zählen ebenfalls der stationäre Handel, der E-Commerce als weiteres Standbein entdeckt, sowie die Hersteller, die nunmehr unter Umgehung des Handels direkt an Endverbraucher liefern können. Bedeutung im grenzüberschreitenden Direktvertrieb dürften vor allem standardisierte Produkte wie Bücher, CDs und Computerhardware haben, mit Einschränkungen aber auch Produkte wie Bekleidung, die (teilweise) für eine individualisierte Massenproduktion geeignet sind.

\section{Organisationales Lernen} und die Umweltleistung

von Unternebmen

und

\section{Homo Sustainomicus?}

sind die Themen der Informationsdienste

\section{Ekologisches Wirtschaften $5 / 02$ und $6 / 02$}

Wenn Sie potenzielle Beiträge haben, wenden Sie sich bitte an die Redaktion.

\section{Onlinehandel als Wachstumsfeld}

Die Mehrzahl der Studien zum B2C-E-Commerce geht für die nächsten Jahre von einem beträchtlichen Umsatzwachstum aus, wenngleich zuletzt die Einschätzungen zurückhaltender wurden. Im Online-Handel sind vor allem Computerhardware, Bücher und CDs/ DVDs, aber auch Geräte der Unterhaltungselektronik, kleine Haushaltsgeräte, Bekleidungs- und Sportartikel, Spielwaren sowie Artikel für die Freizeit von Bedeutung. Gemessen am Gesamtumsatz mit diesen Produkten ist sein Anteil derzeit noch gering: Er wird in Europa auf etwa 0,24 Prozent geschätzt; bei den Hardwareprodukten immerhin auf 3,5, bei Büchern auf 1,6 und bei CDs/ DVDs auf 1,2 Prozent. Für den institutionellen Versandhandel dagegen ist die Relevanz von B2C bereits heute beträchtlich. So gibt der Bundesverband des 
deutschen Versandhandels für seine Mitglieder Online-Anteile an den Bestellungen von bis zu 40 Prozent an (4).

B2C-E-Commerce-Schätzungen für den grenzüberschreitenden Handel gehen immerhin von einem entsprechenden Anteil von 15 bis 20 Prozent am gesamten Online-Handel in den einzelnen europäischen Ländern aus. Noch liegt der grenzüberschreitende Versandhandel in Deutschland bei einem Umsatzanteil von 3,5 Prozent. Die zukünftige Dynamik dürfte stark vom Abbau sprachlicher und kultureller Barrieren sowie von Regelungsdefiziten im Bereich der Zahlungs-, Währungs- und Rechtssysteme abhängen.

\section{- Umweltrelevante Innovations- wirkungen}

Bei Bestehen des Regelungsdefekts haben Direktvertreiber unmittelbare wirtschaftliche Vorteile, da sie die Aufwendungen für die Rücknahme und Verwertung der von ihnen in Verkehr gebrachten Erzeugnisse und Verpackungen bzw. für die Beteiligung an etwaigen Entsorgungssystemen vermeiden können. Soweit sich dadurch marktrelevante Effekte ergeben, wirkt sich dies auf das Verhalten der Unternehmen und des Staates im Empfängerland aus und findet seinen Niederschlag insbesondere in den Innovationsbemühungen sowie bei der Einstellung und Akzeptanz in Hinblick auf die Rahmenbedingungen und das Regulierungsmuster.

Dabei werden sich die Innovationsaktivitäten der Akteure aufgrund des Defekts unterschiedlich verändern. Akteure, die sich ihrer Verantwortung entziehen können, haben suboptimale Anreize zu Innovationen, sodass sie umweltrelevante Eigenschaften ihrer Produkte bzw. Verpackungen nicht hinreichend verbessern. Dies bedeutet beispielsweise, dass in Kraftfahrzeugen eine gesamtwirtschaftlich zu hohe Materialvielfalt enthalten bleibt oder in Elektrogeräten

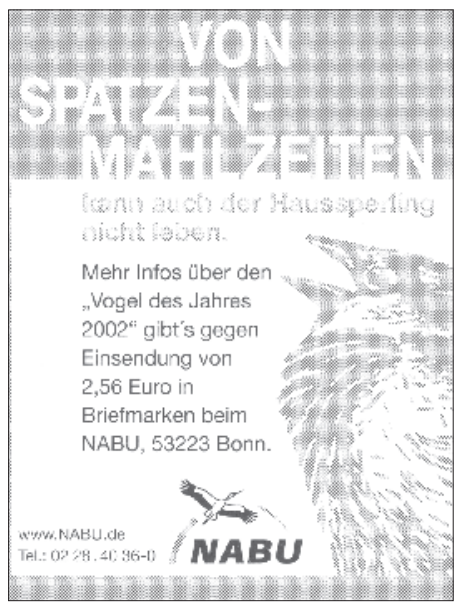

schwer zu entsorgende Stoffe nicht substituiert werden. Dagegen ist denkbar, dass die Akteure, die sich nicht als Freifahrer verhalten (können), sogar gesamtwirtschaftlich überhöhte Innovationsanstrengungen unternehmen, weil sie im Rahmen der kollektiven Wahrnehmung der Produktverantwortung auch für die Entsorgung der Produktabfälle der Hersteller, denen man nicht habhaft wird, verantwortlich zeichnen müssen. Damit verschlechtert sich nicht nur ihre relative Wettbewerbsposition, sie haben auch verstärkte Anreize zum Austritt aus dem kollektiven Entsorgungssystem. Dieses verliert dann an Stabilität und bei Existenz steigender Skalenerträge auch an Effizienz.

Es ist zudem damit zu rechnen, dass bei den inländischen Akteuren die Bereitschaft zur Befolgung der in Rede stehenden Vorschriften erodiert. Insgesamt erscheint die Befürchtung realistisch, dass durch eine derartige Entwicklung die angestrebten ökologischen Wirkungen in dem erhofften Ausmaß ausbleiben.

\section{- Staatliche Reaktionsmöglichkeiten}

In jedem Fall bedarf es seitens des Regelungsgebers einer Anpassung bei den Instrumenten der Produktverantwortung. Grundsätzlich bestehen drei Optionen:

- Herstellung gleicher Wettbewerbsbedingungen durch Vollzug der Vorschriften auch gegenüber ausländischen Direktvertreibern, wobei sich Probleme des Verwaltungsvollzugs ergeben.

- Abmilderung der rechtlichen Anforderungen bis zu einem Grad, bei dem die verbleibende Ungleichbehandlung inländischer und ausländischer Akteure nicht mehr als markrelevant empfunden wird. Dies kann beispielsweise eine Herabsetzung von Verwertungsquoten oder eine Einschränkung der Entsorgungsverantwortlichkeit der hiesigen Hersteller sein.

- Asymmetrischer Vollzug, das heißt Durchsetzung der Vorschriften gegenüber im Inland tätigen Unternehmen unter Inkaufnahme von Ungleichbehandlung und Wettbewerbsverzerrungen gegenüber im Ausland ansässigen Direktvertreibern.

Insbesondere bei der letzten Option ist mit einer Ausweitung des Marktanteils grenzüberschreitend tätiger Direktvertreiber sowie eventuell mit einer Abwanderung von Vertreibern ins Ausland oder der Entstehung entsprechender Umgehungsstrategien zu rechnen. Als Maßnahmen zur Herstellung gleicher Wettbewerbsbedingungen durch öffentlich-rechtliches Handeln innerhalb des Ansatzes der Produktverantwor- tung kommen die Verwaltungshilfe beim Vollzug der Regelungen oder eine Verpflichtung ausländischer Hersteller und Vertreiber, Beiträge an ein im Geltungsbereich der jeweiligen Verordnung bestehendes kollektives Entsorgungssystem abzuführen, infrage. Falls diese Lösungswege nicht gangbar sind, müsste der Einsatz anderer Instrumente außerhalb der Produktverantwortung im Sinne des Kreislaufwirtschaftsgesetzes geprüft werden.

\section{Fazit}

Zwar mag der Anteil der Produkte, die bereits heute oder aber potenziell die Effizienz und Wirksamkeit von Rücknahmeverpflichtungen gefährden, auf dem ersten Blick vergleichsweise gering erscheinen. Dies sollte jedoch angesichts des zu erwartenden internetgestïtzten Wachstums beim grenzüberschreitenden Warenverkehr und vor allem aufgrund der unabhängig von der quantitativen Größenordung bestehenden Regelungsschwächen nicht gering geschätzt werden. Darüber hinaus stellt sich aus juristischer Sicht das Problem, dass der Gleichbehandlungsanspruch untergraben wird.

\section{Anmerkungen}

(1) Clausen, H.: Rücknahmeverpflichtungen als Instrument von Abfallwirtschaftspolitik. Münster 2000.

(2) Den Auswirkungen und möglichen Abhilfen dieses Regelungsdefekts wird im Rahmen des vom BMBF geförderten :[riw]-Projekts „Internalisierung versus Internationalisierung” näher nachgegangen. Dem Projektteam gehören neben den Autorlnnen Dr. Joachim Hafkesbrink und Markus Schroll (ARÖW, Duisburg) sowie Christina Hagengut und Wolfgang Schutt (INTEC Consulting, Bonn) an. Informationen zum Projekt unter www.riw-netzwerk.de und www.aroew.de/inversi/index.html

(3) Müller-Hagedorn, L. (Hrsg.): Zukunftsperspektiven des ECommerce im Handel. Frankfurt/ Main 2000.

(4) U.a. Hauptverband des Deutschen Einzelhandels: HDE-Umfrage eCommerce 2001 (B2C), http://www.einzelhandel.de, vom 06.12.2001; Bundesverband des Deutschen Versandhandels e.V. (Hrsg.): Versandhandel in Deutschland. Frankfurt/ Main 2001.

\section{Die Autorlnnen}

Dr. Hartmut Clausen und Marianne Halstrick-Schwenk sind wissenschaftliche Referenten im Rheinisch-Westfälischen Institut für Wirtschaftsforschung (RWI). Kontakt: RWI, Hohenzollernstr. 1-3, 45128 Essen. Tel. 0201/ 8149-0, Fax -200,

E-Mail: clausen@rwi-essen.de, halst@rwi-essen.de 
(c) 20I0 Authors; licensee IÖW and oekom verlag. This is an article distributed under the terms of the Creative Commons Attribution Non-Commercial No Derivates License (http://creativecommons.org/licenses/by-nc-nd/3.o/), which permits unrestricted use, distribution, and reproduction in any medium, provided the original work is properly cited. 